\title{
CLINICAL STUDY AND COMPARISON OF VARIOUS MODALITIES OF TREATMENT OF VARICOSE VEINS OF LOWER LIMBS
}

\author{
Thatipamula Srinivas ${ }^{1}$, K. Suryanarayana 2 , B. Sharadha ${ }^{3}$, Nyna Sindhu 4 \\ ${ }^{1}$ Assistant Professor, Department of General Surgery, Kakatiya Medical College. \\ ${ }^{2}$ Assistant Professor, Department of General Surgery, Kakatiya Medical College. \\ ${ }^{3}$ Assistant Professor, Department of General Surgery, Kakatiya Medical College. \\ ${ }^{4}$ Final Year Postgraduate, Department of General Surgery, Kakatiya Medical College.
}

\section{ABSTRACT}

\section{BACKGROUND}

Varicose veins of lower limb and their treatment are as old as mankind. Hippocrates discussed them 2500 years ago. In the Indian subcontinent, an estimated $23 \%$ of adults have varicose veins, and $6 \%$ have more advanced chronic venous disease (CVD), including skin changes and healed or active venous ulcers. Most varicose veins are due to primary venous disease. The most frequent cause is likely an intrinsic morphologic or biochemical abnormality in the vein wall, although the aetiology can also be multifactorial.

Aims and Objectives- To study the distribution in age, sex, occupation, clinical features and severity grade of varicose veins of lower limbs. To study the various modalities of management of varicose veins of lower limb effectively and to prevent its complications.

\section{MATERIALS AND METHODS}

The present study is a prospective study conducted after obtaining institutional approval on patients admitted and treated in Mahatma Gandhi Memorial Hospital, Warangal.

\section{RESULTS}

The overall treatment satisfaction was a composite assessment that included treatment deliveries, length of procedure, followup treatment and recovery. It gave an indication of patient acceptability of the procedure.

\section{CONCLUSIONS}

This study revealed that the disease is prevalent in active phase of life and male preponderance, and occupations involving prolonged standing and violent muscular effort are prone to disease. Majority of the patients had long saphenous incompetency and the complications are more when both valvular and perforator systems are involved. Duplex USG is the investigation of choice.

\section{KEYWORDS}

Varicose Veins, Sclerotherapy.

HOW TO CITE THIS ARTICLE: Srinivas T, Suryanarayana K, Sharadha B, et al. Clinical study and comparison of various modalities of treatment of varicose veins of lower limbs. J.Evolution Med. Dent. Sci. 2016;5(85):6304-6309,DOI: 10.14260/jemds/2016/1424

\section{INTRODUCTION}

Varicose veins of lower limb and their treatment are as old as mankind. Hippocrates discussed them 2500 years ago. It is not found in other animals and it is the human beings, who had to pay for their erect posture. Varicose veins constitute a progressive disease that becomes steadily worse. There is never remission of the disease except after pregnancy and delivery, when many of dilated varicosities may disappear. During its course, the disease produces complications, which lead the patient to seek medical care.

Varicose veins, though a common condition many a times remain asymptomatic. It is in the developed countries, where attire reveals more than it conceals, and patients turn up for cosmetic reasons.

Financial or Other, Competing Interest: None.

Submission 21-07-2016, Peer Review 13-08-2016,

Acceptance 20-08-2016, Published 21-10-2016.

Corresponding Author:

Dr. Thatipamula Srinivas,

Flat No. B3, Thirumala Residency,

Naimnagar, KUC Cross Road,

Hanamkonda, Warangal Dist.,

Telangana State.

E-mail: cnoo.dr@gmail.com

DOI: $10.14260 /$ jemds/2016/1424
In our Indian scenario, it is the complications not the cosmetic reasons, bring the patients to the doctor. That is the reason why, though common, varicose veins remain as an iceberg phenomenon.

In the Indian subcontinent, an estimated $23 \%$ of adults have varicose veins, and $6 \%$ have more advanced chronic venous disease (CVD), including skin changes and healed or active venous ulcers. Varicose veins have long been considered a cosmetic problem that only affected emotional well-being but were not the source of disability. Varicosities; however, are frequently the cause of discomfort, pain, loss of working days, disability, and deterioration of health-related quality of life (QOL). Severe CVD may also lead to loss of limb or loss of life.

Evaluation of varicose veins has greatly progressed in the past 2 decades with the widespread availability of duplex ultrasonography. The treatment of varicose veins has also undergone dramatic changes with the introduction of percutaneous endovenous ablation techniques, including endovenous laser therapy (EVLA), radiofrequency ablation (RFA), and liquid or foam sclerotherapy. Open surgical treatment with stripping of the varicose veins performed under general anaesthesia, with the associated pain, potential for wound complications, and loss of working days, has been 
largely replaced by percutaneous office-based procedures that can be performed under local or tumescent anaesthesia with similar early and midterm results but with less discomfort to the patient, improved early QOL, and earlier return to work.

Varicose veins of the lower limbs are dilated subcutaneous veins that are $3 \mathrm{~mm}$ in diameter measured in the upright position. Synonyms include varix, varices, and varicosities.

Varicosity can involve the main axial superficial veins, the great saphenous vein (GSV) or the small saphenous vein (SSV) or any other superficial vein tributaries of the lower limbs.

Most varicose veins are due to primary venous disease. The most frequent cause is likely an intrinsic morphologic or biochemical abnormality in the vein wall, although the aetiology can also be multifactorial. Labropoulos et al ${ }^{1}$ proposed that the origin of venous reflux in patients with primary varicose veins can be local or multifocal structural weakness of the vein wall and that this can occur together or independently of proximal saphenous vein valvular incompetence. Varicosities can also develop as a result of secondary causes, such as previous deep vein thrombosis (DVT), deep venous obstruction ${ }^{2}$, superficial thrombophlebitis, or arteriovenous fistula. Varicose veins may also be congenital and present as a venous malformation.

Varicosities are manifestations of CVD. CVD includes various medical conditions of long duration, all involving morphologic and functional abnormalities of the venous system manifested by symptoms or signs (or both).

\section{AIMS AND OBJECTIVES}

The study of varicose veins in the lower limb has been taken up with two aims.

1. To study the distribution in age, sex, occupation, clinical features and severity grade of varicose veins of lower limbs.

2. To study the various modalities of management of varicose veins of lower limb effectively and to prevent its complications.

\section{MATERIALS AND METHODS}

\section{Study Area}

The present study is a prospective study conducted after obtaining institutional approval on patients admitted and treated in Mahatma Gandhi Memorial Hospital, Warangal which is a Government Hospital, 1000 bedded multispeciality hospital. Our hospital has good load of patients from surrounding villages where the majority of the population is agricultural labourers by occupation.

\section{Study Population}

Patients admitted in our hospital with age group restricted to 18-70 years are studied. Patients who underwent surgeries for the same complaints previously were not included. Informed consent was obtained from all patients and both male and females were included in this study.

\section{Sample Size}

Total numbers of patients studied were 150 (183 limbs). All patients who were admitted and treated are studied and followed up. Patients who were operated for same disease previously and patients with age more than 70 years and less than 18 years were not included.

\section{Data Collection Tools and Techniques}

Data was collected from hospital records and regular followup of the patients was done in OPD.

\section{Inclusion Criteria}

1. All patients admitted with lower limb varicose veins in hospital.

2. Patients aged 18 to 70 years.

3. CEAP: C1-C6.

\section{Exclusion Criteria}

1. Patients aged below 18 and above 70 yrs.

2. Patients in the outpatient department.

3. DVT patients.

4. Anaphylaxis to sclerosants.

5. Bad medical status.

6. Pregnancy status.

7. Infected venous ulcer.

8. Venous malformations.

9. Varicose veins other than lower limb.

10. Medical treatment.

11. Management of leg ulcers.

In our study, Materials Used are-

Sclerosant: STDS (FOAM)

Stripper: Plastic stripper.

Laser: $1020 \mathrm{~nm}$.

Radiofrequency: 120 J per 20 secs.

\section{Case Proforma}

A thorough history was taken in all the patients. A detailed clinical examination was done. All the clinical tests were applied, then all patients were subjected to duplex USG to confirm the diagnosis. The routine investigations were done. The patients underwent treatment based on their clinical and investigational profile. The post-operative course was noted. Further, the patients were followed up. Final outcome evaluated, all the information was taken down in the proforma designed for the study.

\section{RESULTS}

Varicose veins of lower limb is a common clinical manifestation, which starts early in the life but assumes an innocent course for variable length of time.

\section{Sex Distribution}

The male sex appears to be more prone to lower limb varicosities than females. Though the western studies shows the female preponderance, $(81.88 \%$ in Chang C.J. and $73.24 \%$ in AHM study) in present study, $76 \%$ involved are males, only $24 \%$ of patients were females who sought their treatment for complications rather than cosmetic reason. Probably, Indian women cover their body with saree hence they are not much bothered about the appearance of dilated veins.

\section{Age Distribution}

Varicose veins are more common in the age group of 30-50 years. The youngest was 18 years and oldest was 70 years. 
This shows that the disease is more common in younger and active phase of life.

\section{Occupations}

Varicose veins are common in persons whose occupations force them for prolonged standing for long number of hours and violent muscular efforts. In present study, approximately $65 \%$ of the patients are affected by prolonged standing.

\section{Clinical Manifestations}

Varicose veins assume an innocent course for variable length of time. All patients involved in this study had prominent veins. But majority of the patients presented with complications and advanced haemodynamic changes (Oedema, pigmentation \& ulcer) and only $1.33 \%$ of patients presented with only prominent veins. But in western studies, majority of patients were treated for only prominent veins, (54\% in 0'Shaughnessy $\mathrm{M}$ et al) rather than its complications. This shows the cosmetic appearance was the commonest presenting complaint in the western countries, but complications of varicose veins seeks medical attention in India.

\section{Clinical Class of CEAP}

The majority of the patients sought treatment for one or the other complication, $44.26 \%$ patients had class 4 with symptoms of pigmentation with itching and $9.29 \%$ of patients were treated for ulcer. This is very high compared to western studies. In present study, only $1.33 \%$ of patients treated for prominent veins only.

\section{Limb Involvement}

In the present study, the right limb involvement is $30.66 \%$ and left limb involvement is $47.33 \%$. This favourably compares with the study conducted by A.H.M. Dur, A.I.C Mackaay et al. (Right - $43 \%$ and left $-56 \%$ ). The cause for the increased incidence of left side is not known. This is probably because that the left iliac veins join at an angle; being constantly pressed by the loaded left colon, the common iliac artery crossing over the common iliac vein and the longer course traversed by the left iliac veins, unlike the right lower limb is not much subjected to these anatomical disadvantages.

\section{Venous System Involved}

In present study, around $76.5 \%$ of the limbs involved showed the long saphenous involvement and only $4.9 \%$ patients presented with short saphenous involvement. This is favourable with the western studies. This can be explained as the length of long saphenous vein is more, the short saphenous vein runs in fascial tunnel from above the lateral malleolus to the popliteal fossa. The communicating veins are mostly indirect in short saphenous system, while direct communicating veins predominate in the long saphenous system. ${ }^{3}$

\section{Site of Incompetence}

The majority of the patients in this study had combined saphenofemoral and perforator incompetence and only $12 \%$ of patients had isolated perforator incompetence.

\section{Correlation between CEAP classes \& Site on Incompetence}

In present study, the patients with higher grade of clinical class of CEAP had combined valvular incompetence or longstanding saphenofemoral incompetence, this shows that the combined valvular defect and longstanding isolated incompetence with advanced haemodynamic changes are prone to more complications rather than their simple saphenofemoral incompetence. ${ }^{4}$

\section{Duplex USG}

In present study, routine duplex USG was done in all patients preoperatively. Routine preoperative duplex examination leads to improved surgical results and lower recurrence rates.

\section{Management of Varicose Veins}

In our study, $60 \%$ limbs underwent foam sclerotherapy for perforators of all endovenous procedures done for main superficial veins \& for main trunks EVLA for 27.1\%, RFA for $30.5 \%$, flush ligation+ stripping for $31.1 \%$ of limbs.

Lurie et al reported results of the Endovenous Radiofrequency Obliteration (Closure procedure) versus Ligation and Stripping (EVOLVeS) study at 4 months and at 2 years. 5

A recent RCT by Gale et al compared results of $810-\mathrm{nm}$ wavelength laser with RFA. All veins were closed At 1 week after the procedure. The recanalisation rate at 1 year was significantly higher in the RF group (Closure FAST system) than after laser ( 11 of 48 vs. 2 of 46, P 0.002). The mean VCSS score change from baseline to 1 week post-procedure was higher for RFA than EVLA ( $P$ 0.002), but there was no difference between groups at 1 month (P 0.07) and 1 year $(P$ 0.9). The authors concluded that both methods of endovenous ablation effectively reduce symptoms of superficial venous insufficiency. EVLA was associated with greater bruising and discomfort in the perioperative period but may provide a more secure long-term closure than RFA. ${ }^{6}$

After adjusting for duration of follow-up they found that compared with SVS, UGFS and RFA were as effective, and EVLA significantly more effective (van den Bos et al 2009).

Both EVLA and RFA are also considered to be as effective as SVS for the treatment of primary GSV incompetence in terms of early recanalisation rates, and mid-term recurrence of visible VV. Following EVLA, recanalisation rates of $0-9 \%$ at 1-2 years have been reported, (Min et al 2003; Mundy et al 2005; Theivacumar et al 2009, Christenson et al 2010; Pronk et al 2010) although one RCT comparing EVLA and RFA found recanalisation rates of $22 \%(7 / 32)$ and $26 \%$ after treatment (Goode et al 2010). Other studies found recanalisation in 0$19 \%$ of patients treated with RFA at 1 year. (Merchant et al 2002; Merchant et al 2005; Nicolini et al 2005) (9/34) respectively 6 months. ${ }^{7}$

\section{Complications}

In the present study, some minor complications occurred which were managed conservatively. Among these majority of the patients, only 4 limbs had wound infection. The study conducted by Hagmuller GM showed incidence of some major complications none of which occurred in the present study group. 
Only 3 limbs in the present study presented with recurrent varicosities who underwent endovenous procedures and the recurrent varicosities are found to be due to recanalisation and underwent foam sclerotherapy. ${ }^{8}$ This shows the importance of duplex scan for accurate diagnosis of their incompetency to prevent recurrence.

Masuda et al reported clinical results with ultrasonographically guided sclerotherapy using morrhuate sodium in 80 limbs with predominantly perforator incompetence alone. The authors noticed a significant improvement in VCSS, and ulcers rapidly healed in $86.5 \%$, with a mean time to heal of 36 days. The ulcer recurrence rate was $32 \%$ at a mean of 20 months despite low compliance (15\%) with compression hose. New and recurrent perforators were identified in 33\% of limbs, and ulcer recurrence was statistically associated with perforator recurrence.

Guex et al reported early and midterm complications in a prospective multicentre registry that included 12,173 sclerotherapy sessions, consisting of 5434 with liquid, 6395 with foam, and 344 using both. Ultrasonographic guidance was used in 4088 sessions (33.9\%), and 49 incidents or accidents $(0.4 \%)$ occurred, of which 12 were with liquid and 37 with foam. There were 20 cases of visual disturbances, in 19 cases, foam or air block ${ }^{9}$ was used; all resolved shortly, without any after effects. A femoral vein thrombosis was the only severe adverse event in this study, which also demonstrated that sclerotherapy is a safe technique.

In a prospective non-randomised trial, recurrent varicosity was treated by van Groenendael et al in 149 limbs with open surgery and in 67 with EVLA. Wound infections ( $8 \%$ vs. $0 \%$; P .05) and paraesthesias ( $27 \%$ vs. $13 \%$; P .05) were more frequent in the surgery group, whereas the EVLA group reported more perioperative pain or tightness $(17 \%$ vs. $31 \%$; P .05). Hospital stay in the surgery group was longer (P .05) as was the delay before resuming work (7 vs. 2 days; $P$ .0001). At 25 weeks of follow-up, repeat recurrences were reported in $29 \%$ after surgery and in $19 \%$ after EVLA (P $.511)$.

In a prospective study, Van Rij performed Duplex scanning in 377 patients before surgery and then at 2 to 4 weeks and at 6 to 12 months after surgery. Acute DVT was detected in 20 patients (5.3\%). Eight were symptomatic and no PE was observed. Although this series suggests an incidence of DVT that is higher than previously believed, this complication had minimal short-term or long-term clinical significance 20 DVTs, 18 were confined to calf veins, and half of the DVTs had resolved without deep venous reflux (Comparison of Laser, Surgery and foam Sclerotherapy, CLASS study). The incidence of bruising of $13-27 \%$ after RFA (Lurie et al 2003; Vasquez et al 2007) and 11-15\% after EVLA (Rasmussen et al 2007; Christenson et al 2010), while significantly less than after SVS, are still higher than the $7 \%$ rate of bruising after UGFS 10 .

\section{Recurrence}

Recurrent varicose veins. In a 34-year follow-up study of 125 limbs, Fischer et al noted ultrasonographic evidence of saphenofemoral reflux in 75 limbs (60\%). Allegra et al noted a 5 -year recurrence rate of $25 \%$ in a large study that included 1326 patients. Despite technically correct surgery, confirmed with postoperative duplex scanning, recurrence at the SFJ occurred in $13 \%$, at the saphenopopliteal junction in $30 \%$, and at both in $36 \%$.

Creton and Uhl treated 129 limbs with recurrent varicose veins using foam sclerotherapy with $1 \%$ polidocanol combined with surgical treatment. 11 All patients had phlebectomies, and 20 had repeat ligation of the saphenous stumps. Foam sclerotherapy combined with surgery resulted in $93 \%$ closure of the saphenous stumps and no recurrent varicose veins. Two patients had asymptomatic DVT.

\section{Cost Effectiveness}

In practice, the two endothermal treatments (EVLA and RFA) compete directly with each other, and this guideline assumes they have equal clinical effectiveness. The implication of this is that, in this analysis, whichever of these two treatments is the cheapest will be cost-effective. However, due to uncertainty around the costs, specifically that the RFA catheter is more expensive, but the EVLA requires a laser controlled area, it is not straight forward to identify which of these treatments is cheaper. For the purpose of this analysis it was assumed that once the laser controlled area had been accounted for, EVLA would cost no more than RFA; the cost of RFA was therefore assumed to be the maximum cost of endothermal treatment. This maximum cost was used in the model base case, and costs were explored thoroughly through sensitivity analysis.

On the other hand, patient expectations are usually higher if the cost is higher, which influence satisfaction and therefore QoL scores after treatment. This may explain for the non-significant difference in QoL scores between RFA and HS, since the degree of symptomatic improvement after RFA may be counteracted by the higher cost and expectation from the patient. Considering the similar QoL scores and recurrence rates in our study, the possible benefits reported by other studies may not be enough to overcome the higher costs, although a cost-benefit analysis may be needed to further clarify this issue.

\section{Quality of Life}

The efficacy of foam sclerotherapy on QOL was recently demonstrated in a single-centre cohort study by Darvall et al. These author found that ultrasound-guided foam sclerotherapy for great and small saphenous varicose veins leads to significant improvements in generic and diseasespecific health related QOL for at least 12 months after treatment 12 .

Marked improvement in QOL after open surgery was also demonstrated in an RCT by Rasmussen et al.

In a prospective cohort study, Mackenzie et al monitored 102 consecutive patients who underwent varicose vein surgery. At 2 years after surgery, health-related QOL markedly improved to baseline when assessed with the Aberdeen ${ }^{13}$.

Varicose Vein Symptom Severity Score and the SF-36. Improvement in QOL from varicose vein surgery has been shown to be statistically significant and clinically meaningful, matching the benefits observed after elective laparoscopic cholecystectomy.

In terms of QoL, Subramonia and Lees. ${ }^{14}$ showed an overall better improvement in QoL after RFA in the shortterm, although different types of questionnaires showed different results. The EVOLVeS study reported a progressive 
decrease in QoL score change between 1 week and 4 months, but the differences reappeared at 1 year and remained significant at 2 years after treatment, showing long-term advantages of RFA. ${ }^{15}$ On the other hand, Rautio et al showed that there was no difference in QoL scores between the two groups.

This study has the limitation of being a randomised study performed in a single institution and therefore selection bias may have affected the results, which makes direct comparison with previously published randomised control trials inadequate. Patient or doctor preference may have influenced the choice of treatment; however, the baseline characteristics were only different for sex, with the female predominance for RFA reflecting their willingness for better cosmetic results. However, the difference in cost makes a randomised control trial difficult to be performed.

\section{Also Randomised Control Trials have the Disadvantage of having Smaller Numbers Overall}

- This study revealed that the disease is prevalent in active phase of life and male preponderance.

- Occupations involving prolonged standing and violent muscular effort are prone to the disease.

- Majority of the patients had long saphenous incompetency and the complications are more when both valvular and perforator systems are involved.

- Duplex USG is the investigation of choice.

- Accurate preoperative evaluation and ligation of site of incompetency are key to success.

- Important changes have been adopted in the classification, scoring and anatomical notation of varicose veins in the past few years.

- A quality of life or degree of disability assessment is an important part of the initial consultation.

- Many patients simply require reassurance, and a thorough discussion of options at the primary care level may circumvent unnecessary referral.

- Compression stockings alone may be appropriate for patients who are too unfit for intervention or those who do not wish to have any form of surgical intervention.

- Many patients are treated for cosmetic concerns alone, so it is important to manage patients' expectations.

- Minimally invasive treatment options such as injection sclerotherapy and endovenous modalities are becoming increasingly popular and have shown equivalence in short-term outcomes.

- Conventional open surgery has also improved, with better outcomes, smaller incisions and duplex mapping.

- RF ablation is associated with less pronounced postprocedural pain syndrome compared with EVLA.

- Ablation rate and recanalisation rate for the target vein are not significantly different by 5 months follow-up among endovenous procedures

- Pronounced improvement of QOL scores and clinical severity scores was noted after treatment with RF ablation compared with EVLA procedure, though clinical significance of this difference is quite low

- 175 Patients have been treated and analysed. 118 Patients (67\%) underwent endovenous laser ablation, 57 patients (33\%) underwent ligation of the SPJ. Both treatment modalities reduced pain after 6 weeks. One week post-treatment patients in the EVLA-group temporarily experienced more pain compared to the surgery-group (31 vs. 18 on a VAS of $0-100$ ). There were no significant differences between the two groups with respect to quality of life. Both treatments did show improvement in quality of life.

- EVLA provides an excellent alternative to conventional surgery in the treatment of symptomatic varicosis due to an incompetent GSV with SFJ. EVLA has a superior immediate success rate, easier, faster and has fewer complications.

- Endothermal treatment is likely to be the cost-effective treatment strategy for bilateral treatment.

- An area of particular uncertainty was the costs, yet sensitivity analyses revealed that the model is robust to changes in relative costs. For example, even if the differences in costs have been underestimated, endothermal treatment would remain the optimal strategy even if the costs of all the other treatments are half of what we estimated in the base case. If the costs of surgery, sclerotherapy and conservative care remain as specified in the base case, endothermal treatment remains cost effective even with increases in cost.

- The benefit of compression after interventional treatment for varicose veins is unclear.

- EVLA and surgery provide similar Quality of Life and clinical improvements in patients with varicose veins. Standard surgical treatment of varicose veins; however, is associated with QoL limitations in the early postoperative period. EVLA has been shown to remove the QoL limitations experienced by patients in the early surgical postoperative period.

- In deliverance of modern day surgical services, the trend towards less invasive interventions is clearly obvious. This is driven by patient, surgeon, and hospital management preferences, (possibly) imposed by the current economic climate. The hope (or assumption) is that the less invasive interventions would lead to a reduction in complications, length of hospital stay, and cost. The delivery of varicose vein services has towed the same line as the rest of surgery.

- As already alluded to, not every patient or every varicose vein will be suitable for endovenous ablation; therefore surgery would still play an important role in management of varicose veins. The growth in the use of foam sclerotherapy, means that there is yet another tool for the treatment of suitable veins and patients.

- There is as yet no one-cap-fits-all modality, and although almost any varicose vein can be treated by surgery, not all patients will want to have, (nor indeed can be candidates to have) open surgery. In spite of its current status as gold standard, it is inevitable that the role of standard surgery in the treatment of lower limb varicose vein will shrink significantly in the nearest future, in line with the expansion of minimally invasive techniques.

\section{REFERENCES}

1. Kaplan RM, Criqui MH, Denenberg JO, et al. Quality of life in patients with chronic venous disease: San Diego population study. J Vasc Surg 2003;37(5):1047-53.

2. Smith JJ, Guest MG, Greenhalgh RM, et al. Measuring the quality of life in patients with venous ulcers. J Vasc Surg 2000;31(4):642-9. 
3. Smith JJ, Garratt AM, Guest M, et al. Evaluating and improving health-related quality of life in patients with varicose veins. J Vasc Surg 1999;30(4):710-9.

4. Korn P, Patel ST, Heller JA, et al. Why insurers should reimburse for compression stockings in patients with chronic venous stasis. J Vasc Surg 2002;35(5):950-7.

5. Coleridge-Smith P, Labropoulos N, Partsch H, et al. Duplex ultrasound investigation of the veins in chronic venous disease of the lower limbs: UIP consensus document: part I. Basic principles. Eur J Vasc Endovasc Surg 2006;31 (1): 83-92.

6. Hoggan BL, Cameron AL, Maddern GJ. Systematic review of endovenous laser therapy versus surgery for the treatment of saphenous varicose veins. Ann Vasc Surg 2009;23(2):277-87.

7. Mundy L, Merlin TL, Fitridge RA, et al. Systematic review of endovenous laser treatment for varicose veins. Br J Surg 2005;92(10):1189-94.

8. Luebke T, Gawenda M, Heckenkamp J, et al. Meta-analysis of endovenous radiofrequency obliteration of the great saphenous vein in primary varicosis. J Endovasc Ther 2008;15(2):213-23.
9. Luebke T, Brunkwall J. Systematic review and metaanalysis of endovenous radiofrequency obliteration, endovenous laser therapy, and foam sclerotherapy for primary varicosis. J Cadiovasc Surg (Torino) 2008;49(2): 213-33.

10. Jia X, Mowatt G, Burr JM, et al. Systematic review of foam sclerotherapy for varicose veins. Br J Surg 2007;94(8): 925-36.

11. Leopardi D, Hoggan BL, Fitridge RA, et al. Systematic review of treatments for varicose veins. Ann Vasc Surg 2009;23(2):264-76.

12. Murad MH, Coto-Yglesias F, Zumaeta-Garcia M, et al. A systematic review and meta-analysis of the treatments of varicose veins. J Vasc Surg 2011;53(5 Suppl):49S-65S.

13. Meissner MH, Gloviczki P, Bergan J, et al. Primary chronic venous disorders. J Vasc Surg 2007;46(Suppl S):54S-67S.

14. Kistner RL, Eklof B. Classification and etiology of chronic venous disease. In: Gloviczki P, ed. Handbook of venous disorders: guide-lines of the American venous forum. $3^{\text {rd }}$ edn. London: Hodder Arnold 2009:37-46.

15. Labropoulos N, Giannoukas AD, Delis K, et al. Where does venous reflux start? J Vasc Surg 1997;26(5):736-42. 